\title{
Modelling of solar explosive events in 2D environments
}

\section{Observable consequences}

\author{
I. Roussev ${ }^{1,2}$, J. G. Doyle ${ }^{1}$, K. Galsgaard ${ }^{3}$, and R. Erdélyi ${ }^{4}$ \\ 1 Armagh Observatory, College Hill, Armagh, BT61 9DG, N. Ireland \\ e-mail: jgd@star.arm.ac.uk \\ 2 High Altitude Observatory, NCAR, 3450 Mitchell Lane, Boulder, CO 80301, USA \\ 3 School of Mathematics \& Statistics, Univ. of St. Andrews, St. Andrews, Fife KY16 9SS \\ e-mail: klaus@mcs.st-and.ac.uk \\ 4 Space \& Atmosphere Research Center, Univ. of Sheffield, Hounsfield Road, Sheffield, S3 7RH \\ e-mail: Robertus@sheffield.ac.uk
}

Received 28 May 2001 / Accepted 25 October 2001

\begin{abstract}
We examine the response via line synthesis of two representative transition region lines, namely C IV $1548.2 \AA$ and O vi $1031.9 \AA$, in various physical environments representing the "quiet" Sun to magnetic reconnection events. Our calculations of ion populations allow for departures from equilibrium ionization (EI), which is critical in studies of transient events. Both lines reveal highly blue- and red-shifted Doppler components, and some of the results are examined in the context of solar explosive events. The observable consequences of magnetic reconnection are subtle in the various physical circumstances examined here and differ from one emission line to another, because of the difference in their formation temperatures.
\end{abstract}

Key words. MHD - Sun: atmosphere - Sun: chromosphere - Sun: UV radiation - Sun: magnetic fields line: profiles

\section{Introduction}

Recently, Roussev et al. (2001a) explored the response of a $2 \mathrm{D}$ physical environment approximating the solar transition region to a magnetic reconnection event, and computed time-series in the resonance transition region lines of $\mathrm{C}$ IV and $\mathrm{O}$ VI considering non-equilibrium ionization. The examined model situation was relevant to solar explosive events and involved 2D dissipative MHD. Effects of the non-linear field-aligned thermal conduction, radiative losses, and volumetric heating were also taken into account. Furthermore, a comparison between the dynamic response of various physical circumstances representing the "quiet" Sun to a magnetic reconnection event was made by Roussev et al. (2001b). It was found that the dynamic time-scales involved in the four different experiments presented there are strongly dependent on the initial physical state, although the general dynamics of the reconnection problem is similar.

The present work is a continuation of those two studies and is dedicated to compare the observable signatures of reconnection events in various physical circumstances. All

Send offprint requests to: I. Roussev,

e-mail: ilr@star.arm.ac.uk the computations are based on the MHD results presented in earlier studies and involve the necessary consideration of non-equilibrium ionization (Non-EI).

We aim to accomplish two main goals. First, we discuss the departures from the equilibrium ionization, and consequently justify the necessity of considering nonequilibrium ionization in numerical studies of relevance to transient events. Results on this part of our study are summarized in Sect. 3. As outlined in Sect. 4, the second goal is to synthesize resonance transitions of Li-like ions, namely C IV 1548.2 $\AA$ and O VI $1031.9 \AA$, as well as discuss what model situation(s) among the four examined would best confirm the observational signatures of solar explosive events. The line synthesis in the $\mathrm{C}$ IV line is presented in Sect. 4.1, while that in O VI is discussed in Sect. 4.2. A summary of the results is made in Sect. 5 .

\section{Description of various initial setups}

We again consider the same four physical situations as those discussed in our previous studies (see Roussev et al. 2001a,b). They represent the following cases:

- presence of a transition region and a density enhancement in the current concentration (A1); 
- presence of a transition region and a uniform mass density across the current concentration (A2);

- no transition region and the presence of enhanced mass density in the current concentration (B1);

- no transition region and a uniform mass density across the current concentration (B2).

In order to be consistent and refer to the relevant parameter setup chosen for each particular case, here we use the same abbreviations as those made in Roussev et al. (2001b), i.e., "A1", "A2", "B1", and "B2", respectively.

The line synthesis presented here is done in $1 \mathrm{D}$, along a ray lying on the jet symmetry axis, $x^{*}=0$, where the maximum jet velocities occur (see Roussev et al. 2001a,b). The time-dependent ion populations of carbon and oxygen are calculated using as an input the 2D MHD solutions averaged over 7 grid-points with respect to the $x^{*}$-coordinate. This is done in a symmetrical manner with respect to $x^{*}=0$. Thus, the only velocity component of importance is that parallel to the jet symmetry axis.

Since the C IV 1548.2 $\AA$ and O VI 1031.9 $\AA$ resonance lines have different temperatures of formation, it was necessary to perform a few more experiments with the particular parameter setup of experiments "A1", "A2", and "B2", but choosing as a normalization unit of temperature $2 \times 10^{5} \mathrm{~K}, 5 \times 10^{4} \mathrm{~K}$, and $3 \times 10^{4} \mathrm{~K}$, respectively. This was done for the purpose of having a variety of MHD solutions at different initial temperatures to make the jets "show up" in C IV and O VI. Note this affected only the normalization units, but did not change any value of the model parameters of a particular experiment setup. For instance, if the value of temperature is decreased by a factor of two, then the value of the mass density is increased by the same factor, while the Alfvén speed is decreased by a factor of $\sqrt{2}$. All these changes leave the plasma "beta" parameter unchanged, and therefore the strength of the magnetic field is the same since the kinetic gas pressure is unchanged. This scaling, however, affects the radiative losses, the speed of the thermal conduction, and also the background heating rate. However, as inferred in Roussev et al. (2001b), these taken together are of minor importance for the dynamics of the reconnection events.

A listing of the normalization units adopted in the four MHD experiments relevant to the line synthesis in the C IV line is given in Table 1. The last row in this table gives the current time in absolute units at which the time series in the $\mathrm{C}$ IV line end. As a reference experiment we choose the one presented in Roussev et al. (2001a). This corresponds to the particular setup of experiment "A1".

Similarly, in Table 2 are given the normalization units chosen for the four MHD experiments relevant to the line synthesis in the $\mathrm{O}$ vi line. Here again the last row in this table shows the current time in absolute units at which the time series in the $\mathrm{O}$ vi line finish. As a reference experiment we again take that presented in Roussev et al. (2001a), and relevant to experiment "A1".
Table 1. Set of experiments used to perform line synthesis in the $\mathrm{C}$ IV line.

\begin{tabular}{|c|c|c|c|c|c|}
\hline Physical Value & Notation & A1 & A2 & B1 & B2 \\
\hline $\begin{array}{l}\text { Length } \\
\left(10^{8} \mathrm{~cm}\right)\end{array}$ & 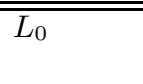 & 3 & 3 & 3 & 3 \\
\hline $\begin{array}{l}\text { Alfvén Velocity } \\
\left(10^{7} \mathrm{~cm} / \mathrm{s}\right)\end{array}$ & $v_{\mathrm{A} 0}$ & 1.293 & 0.915 & 1.293 & 0.709 \\
\hline Time $^{a}(\mathrm{~s})$ & $t_{\mathrm{A} 0}$ & 23.2 & 32.81 & 23.2 & 42.36 \\
\hline $\begin{array}{l}\text { Density } \\
\left(10^{-14} \mathrm{~g} / \mathrm{cm}^{3}\right)\end{array}$ & $\rho_{0}$ & 5.019 & 10.038 & 5.019 & 17.30 \\
\hline $\begin{array}{l}\text { Temperature } \\
\left(10^{5} \mathrm{~K}\right)\end{array}$ & $T_{0}$ & 1 & 0.5 & 1 & 0.3 \\
\hline End Time (s) & $t_{\text {end }}$ & 141 & 157 & 131 & 177 \\
\hline
\end{tabular}

${ }^{a}$ Note that $t_{\mathrm{A} 0}=L_{0} / v_{\mathrm{A} 0}$.

Table 2. Set of experiments used to perform line synthesis in the $\mathrm{O}$ vi line.

\begin{tabular}{|c|c|c|c|c|c|}
\hline Physical Value & Notation & $\mathrm{A} 1$ & $\mathrm{~A} 2$ & $\mathrm{~B} 1$ & $\mathrm{~B} 2$ \\
\hline $\begin{array}{l}\text { Length } \\
\left(10^{8} \mathrm{~cm}\right)\end{array}$ & $L_{0}$ & 3 & 3 & 3 & 3 \\
\hline $\begin{array}{l}\text { Alfvén Velocity } \\
\left(10^{7} \mathrm{~cm} / \mathrm{s}\right)\end{array}$ & $v_{\mathrm{A} 0}$ & 1.829 & 1.293 & 1.293 & 0.915 \\
\hline Time (s) & $t_{\mathrm{A} 0}$ & 16.5 & 23.2 & 23.2 & 32.81 \\
\hline $\begin{array}{l}\text { Density } \\
\left(10^{-14} \mathrm{~g} / \mathrm{cm}^{3}\right)\end{array}$ & $\rho_{0}$ & 2.510 & 5.019 & 5.019 & 10.38 \\
\hline $\begin{array}{l}\text { Temperature } \\
\left(10^{5} \mathrm{~K}\right)\end{array}$ & $T_{0}$ & 2 & 1 & 1 & 0.5 \\
\hline End Time (s) & $t_{\text {end }}$ & 99 & 110 & 131 & 137 \\
\hline
\end{tabular}

\section{Departures from equilibrium ionization}

As pointed out in Roussev et al. (2001a), the equilibrium ionization is a valid approximation only when temperature changes in the emitting plasma take place on time-scales greater than the ionization and recombination times. In such circumstances, the plasma would have sufficient time to readjust the relative ion populations to the new temperature reached. This is no longer the case, however, when bulk motions and gradients in the emitting plasma produce dynamic time-scales short compared to the ionization and recombination times. Hence, in order to describe this physical situation more precisely, time-dependent ion populations need to be calculated.

For a given ionization stage $r$, the rate at which the ion number density, $N_{r}$, changes with time is described by

$$
\begin{aligned}
\frac{\mathrm{D} N_{r}}{\mathrm{D} t} \equiv & \frac{\partial N_{r}}{\partial t}+v_{x} \cdot \frac{\partial N_{r}}{\partial x}+v_{y} \cdot \frac{\partial N_{r}}{\partial y} \\
= & N_{\mathrm{e}}\left[N_{r-1} S_{r-1}+N_{r+1}\left(\alpha_{r+1}^{(\mathrm{r})}+\alpha_{r+1}^{(\mathrm{d})}\right)\right] \\
& -N_{\mathrm{e}} N_{r}\left(S_{r}+\alpha_{r}^{(\mathrm{r})}+\alpha_{r}^{(\mathrm{d})}\right) .
\end{aligned}
$$

Here $\alpha_{r}^{(\mathrm{r})}, \alpha_{r}^{(\mathrm{d})}$, and $S_{r}$ are the rate coefficients of radiative recombination, di-electronic recombination, and collisional ionization for ionization stage $r$, respectively. In the case of equilibrium ionization (EI hereafter), 
the right-hand side of Eq. (1) is equal to zero, i.e., $\mathrm{D} N_{r} / \mathrm{D} t=0$, and thus the above equation reduces to

$$
\begin{aligned}
N_{r}\left(S_{r}+\alpha_{r}^{(\mathrm{r})}+\alpha_{r}^{(\mathrm{d})}\right)= & N_{r+1}\left(\alpha_{r+1}^{(\mathrm{r})}+\alpha_{r+1}^{(\mathrm{d})}\right) \\
& +N_{r-1} S_{r-1} .
\end{aligned}
$$

In this case, the ionization balance is controlled mainly by the electron temperature and only very weakly depends on the electron number density, $N_{\mathrm{e}}$ (see Summers 1972).

This is, however, very unlikely to be the physical situation encountered with those reconnection events. In such circumstances, time-dependent ion populations described by Eq. (1) must be considered. In the present study, the ion populations of carbon and oxygen are computed using a 1D "non-equilibrium ionization" (Non-EI) code developed for the purpose of solving Eq. (1), based on an adaptive grid algorithm (see Sarro et al. 1999), where $\left|v_{x} \cdot \partial N_{r} / \partial x\right|=0$ by assumption. The ionization and recombination coefficients adopted in this code are those given by Arnaud \& Rothenflug (1985). We performed fifthorder polynomial fits to these coefficients in order to get smooth functions of temperature. This code is also based on the assumption of a Maxwellian electron energy distribution. By knowing the values of $v_{y}, N_{\mathrm{e}}$, and $T$ provided by the MHD solutions, one can compute the ion populations of a given element at any stage of the MHD simulations.

In the following, we do not intend to analyze in depth the complicated physics involved in causing departures from EI. Instead, we only show that the approximation of EI does not hold true, which proves necessary the consideration of non-equilibrium ionization.

Let us denote the relative ion populations of $\mathrm{C}$ IV and $\mathrm{O}$ VI by $N_{\mathrm{CIV}} / N_{\mathrm{C}}$ and $N_{\mathrm{OVI}} / N_{\mathrm{O}}$, respectively. Figure 1 shows $N_{\text {CIV }} / N_{\mathrm{C}}(y, t)$ (upper panels), and $N_{\text {OVI }} / N_{\mathrm{O}}(y, t)$ (lower panels), as a function of position along the line-ofsight, $y / L_{0}$ (also $y^{*}$ ), and physical time, $t$, in the case of EI (left panels) and Non-EI (right panels), respectively. Figure 1 consists of image plots, where different degrees of gray in a gray-scale palette represent different values of the relative ion populations, as shown in the gray-scale bars attached. For a better comparison, the upper, respectively lower, panels are scaled in the same dynamic range. The $X$-point in both experiments is located at $y_{0}^{*}=-0.5$, and therefore the initial peak values of the relative ion populations occur at some $y^{*}>y_{0}^{*}$, since the initial background temperature increases in the positive $y^{*}$-direction along the line-of-sight. It is important to note that the initial populations of both oxygen and carbon used to run the "Non-EI" code are calculated assuming EI, i.e., $N_{\mathrm{CIV}} / N_{\mathrm{C}}(y, 0)^{\mathrm{Non}-\mathrm{EI}}=N_{\mathrm{CIV}} / N_{\mathrm{C}}(y, 0)^{\mathrm{EI}}$, and $N_{\mathrm{OVI}} / N_{\mathrm{O}}(y, 0)^{\mathrm{Non}-\mathrm{EI}}=N_{\mathrm{OVI}} / N_{\mathrm{O}}(y, 0)^{\mathrm{EI}}$, consistent with the equilibrium assumptions made for the initial state.

Once the reconnection process starts taking place, it is to be expected that departures from EI will occur at the locations with the fastest moving plasma or/and where the most rapid changes in the physical conditions occur. There are apparent differences between the left and right panels in Fig. 1. For both ions, the relevant populations in the case of Non-EI are mostly greater than those in EI. In the first place, this is found to be at the expense of one ionization stage lower for both carbon and oxygen, namely C III and $\mathrm{O} \mathrm{V}$, respectively, as becoming de-populated. There are also locations, however, where the time-dependent ion populations are lower that the relevant equilibrium values. This is found to be at the benefit of one ionization stage higher for both carbon and oxygen, namely $\mathrm{C} \mathrm{V}$ and O VII, respectively, as becoming more populated. All this occurs at the locations where the plasma is being heated on a relatively short dynamic time-scale, or flows with a high speed into a hotter region, mostly concerning the "blue" jets. (The "blue" jet is the reconnection jet moving in the positive $y^{*}$-direction towards the hightemperature region). At the locations where the recombination rate coefficients become relatively more important than the ionization ones, the time-dependent increase of $\mathrm{C}$ IV and $\mathrm{O}$ VI ion populations occur at the expense of one ionization stage higher, namely $\mathrm{C} \mathrm{V}$ and $\mathrm{O}$ VII, respectively, as becoming de-populated. It is the radiative recombination which is important in this process. This occurs, for example, when the plasma is being rapidly cooled down, or flows into a cooler region. The latter concerns the plasma inside the "red" jets.

The biggest departures from EI for the C IV ion are seen at the end of the "A1" experiment, and occur at $y^{*} \approx 0.9$. Similarly, for the $\mathrm{O}$ VI ion the maximum departures from EI are observed at time $t \approx 92 \mathrm{~s}$, and appear at $y^{*} \approx-0.1$. One result is clear from this analysis. Departures from EI do take place, and therefore Non-EI has to be considered in order to calculate the corresponding ion populations of carbon and oxygen in all experiments more precisely.

\section{Line synthesis}

Once the time-dependent ion populations are computed, the emissivity in a given resonance line per unit interval of wavelength is proportional to

$\varepsilon_{\lambda} \propto \frac{h c}{\lambda} \frac{\Upsilon}{\omega} \frac{N_{1}}{N_{\text {ion }}} \frac{N_{\text {ion }}}{N_{\mathrm{el}}} \frac{N_{\mathrm{el}}}{N_{\mathrm{H}}} N_{\mathrm{H}} N_{\mathrm{e}} \frac{\Psi(\lambda)}{\sqrt{T}} \exp \left(-\frac{W}{k_{\mathrm{B}} T}\right)$.

This is valid only for optically thin and collisionally excited resonance lines. Here $h$ is the Planck constant, $c$ is the speed of light, $\Upsilon(T)$ is the Maxwellian averaged collisional strength, $\omega$ is the statistical weight of the lower level, $N_{1} / N_{\text {ion }}$ is the ratio of ions responsible for the emission in the ground state relative to the total number of ions per unit volume, $N_{\text {ion }} / N_{\mathrm{el}}(y, t)$ is the relative population of the ion, $N_{\mathrm{el}} / N_{\mathrm{H}}$ is the element abundance, $N_{\mathrm{H}}$ is the proton number density, $N_{\mathrm{e}}$ is the electron number density, $W$ is the energy difference between the upper and lower levels, $k_{\mathrm{B}}$ is the Boltzmann constant, and $T$ is the temperature. Also, $\Psi(\lambda)$ is the line profile function given by

$\Psi(\lambda)=\frac{1}{\sqrt{\pi}} \frac{1}{\Delta \lambda_{\mathrm{D}}} \exp \left[-\left(\frac{\lambda-\lambda_{0}-\lambda_{\mathrm{S}}}{\Delta \lambda_{\mathrm{D}}}\right)^{2}\right]$ 

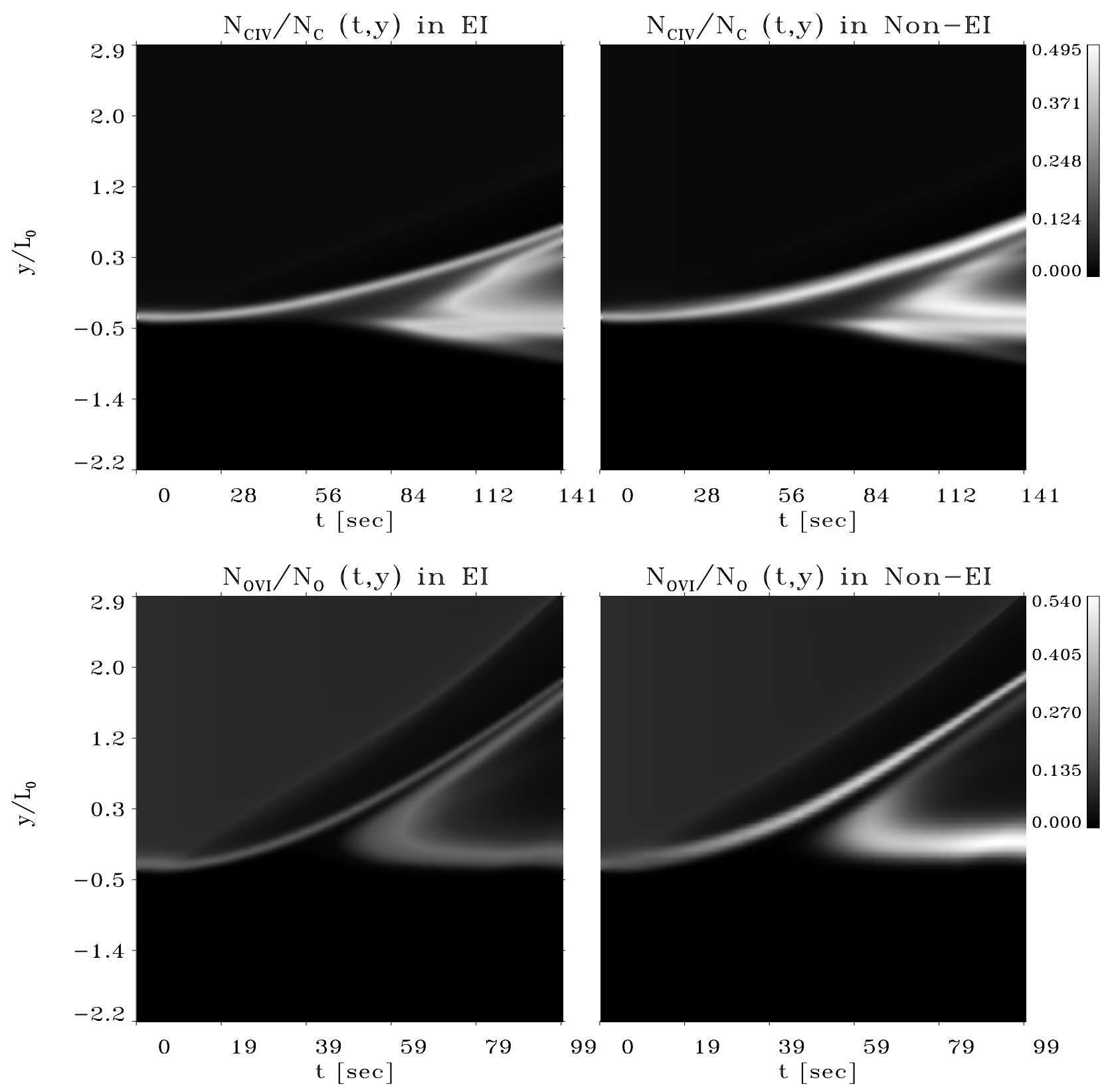

Fig. 1. Relative ion populations of C IV (upper panels) and O VI, respectively, as functions of position along the line-of-sight, $y / L_{0}$, and physical time, $t$. These are shown in the case of EI (left panels) and Non-EI, respectively, and refer to experiments "A1" (see text for more details).

Here $\lambda_{0}$ is the rest wavelength of the resonance line and $\lambda_{\mathrm{S}}=\lambda_{0} v_{\mathrm{p}} / c$ is the Doppler-shift corresponding to plasma velocity of bulk motion, $v_{\mathrm{p}}$, projected on the line-of-sight. The Doppler-width of the emission line, $\Delta \lambda_{\mathrm{D}}$, is explicitly given by

$$
\Delta \lambda_{\mathrm{D}}=\frac{\lambda_{0}}{c} \sqrt{\frac{2 k_{\mathrm{B}} T}{m_{\mathrm{i}}}},
$$

where $m_{\mathrm{i}}$ is the mass of the ion emitting in the resonance line of interest. Here, we consider thermal broadening only and ignore the presence of any micro-turbulence in the emitting plasma.
Once knowing the distribution of emissivities, $\varepsilon_{\lambda}(y, t)$, along the line-of-sight, the line intensity can be computed from

$I_{\lambda}(t)=\int_{-y_{\max }}^{y_{\max }} \varepsilon_{\lambda}\left(y^{\prime}, t\right) \mathrm{d} y^{\prime}$,

where $2 y_{\max }$ is the total length involved in the emission.

\subsection{Time series in the CIV 1548.2 A resonance line}

Line profiles were computed every $0.5 \mathrm{~s}$. The last row in Table 1 gives the time in physical units at which the time-series for each experiment end. Figure 2 presents the 

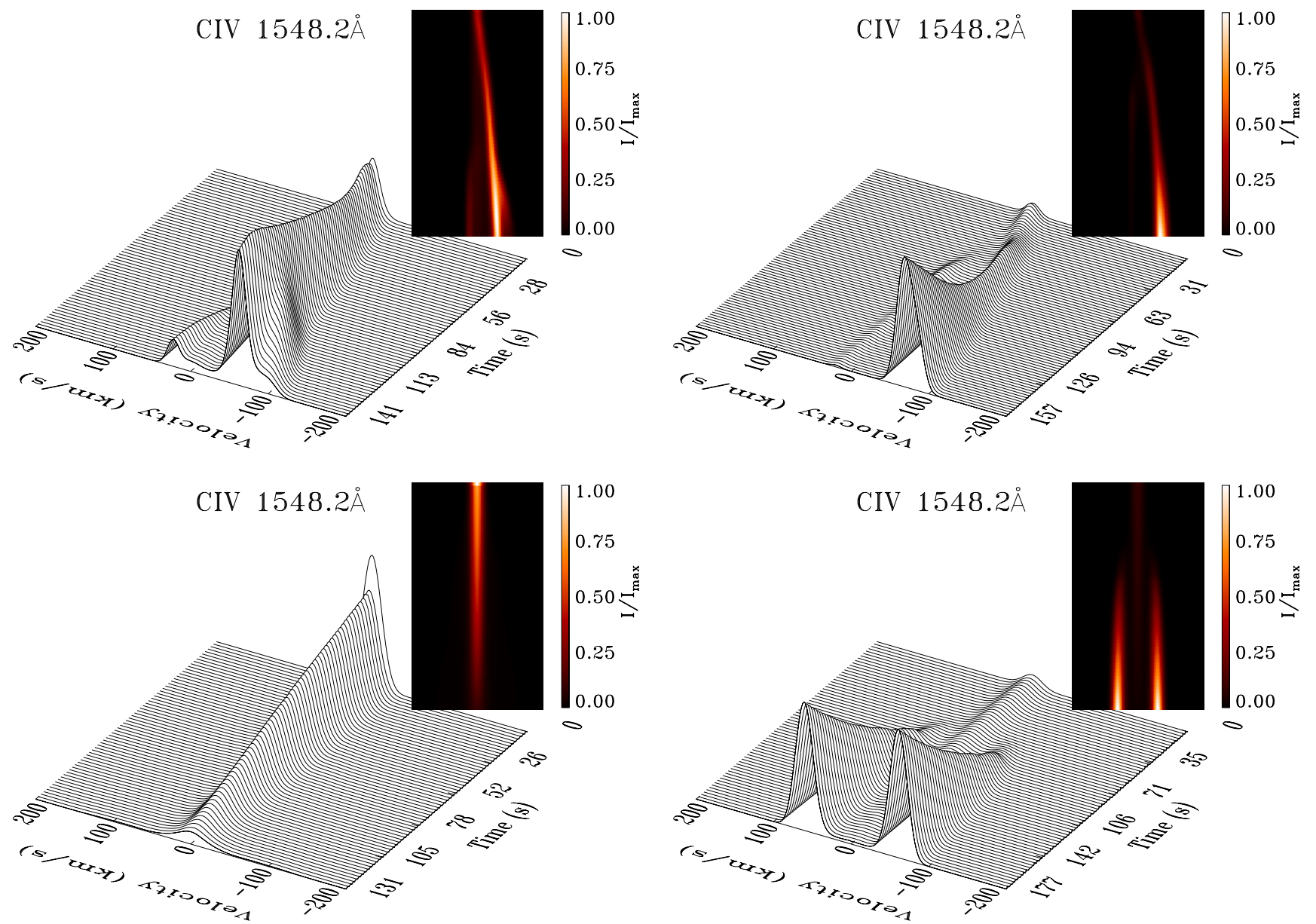

Fig. 2. Line synthesis shown as time-series in the C IV $1548.2 \AA$ resonance line. The upper panels refer to experiments "A1" (left) and "A2", while the lower ones represent experiments "B1" (left) and "B2", respectively. The adjacent image on each panel shows the time-evolution of the spectral line intensity. Note that the time in these images goes from top to bottom (see text for more details).

results obtained from the line synthesis in the $\mathrm{C}$ IV line. The upper panels in this figure refer to experiments "A1" (left) and "A2", while the lower ones represent experiments "B1" (left) and "B2", respectively. The line profiles are plotted as a function of Doppler-shift, $v_{\mathrm{p}}=c \lambda_{\mathrm{S}} / \lambda_{0}$, instead of wavelength. Negative velocities correspond to blue-shifts. Note that the time in the surface plots goes from right to left, and therefore the last line profile in the time-series appears in the foreground. The adjacent image on each panel shows the time-evolution of the spectral line intensity in a gray-scale palette. Note that the time in these images goes from top to bottom, while the Doppler velocity increases from right to left.

In the "A1" experiment, the initial temperature at the $X$-point (not to be confused with the reference temperature, $T_{0}$ ) is approximately half the equilibrium temperature of formation of the resonance $\mathrm{C}$ IV line. The 2D MHD results obtained for this case show the development of two outflow jets: one propagating towards the hightemperature region ("blue" jet), and another one moving in the opposite direction ("red" jet). In such circumstances, one would expect to "observe" in the resonance line of C IV mainly the "blue" outflow jet, since the initial peak population of $\mathrm{C}$ IV is reached somewhere in the hightemperature region and away from the $X$-point. The corresponding time-series in the $\mathrm{C}$ IV line show a rapid decrease in the central line intensity (at zero value of Dopplershift), while a blue-shifted component gradually develops, which at one stage becomes dominant in the spectra. As time passes, this Doppler-shifted component appears at both increased intensity and value of the blue-shift. On the surface plot, it can be seen that there is a faint redshifted component which occurs with gradually increasing intensity. The blue-shifted component, however, evolves much faster than the red-shifted component and undergoes more substantial changes in intensity. At a later stage, after $t \approx 100 \mathrm{~s}$, one can notice the occurrence of another blue-shifted component. This appears at higher Doppler velocity than the main blue-shifted component, but at lower relative intensity. At the end of the timeseries, all these components are present in the spectra and the largest value of the blue-shift is $-120 \mathrm{~km} \mathrm{~s}^{-1}$. The maximum value of the red-shift, however, is much smaller $\sim 40 \mathrm{~km} \mathrm{~s}^{-1}$. Note that the line-core emission is still present in the spectra, although visible at very low relative intensity. The most dominant intensity component 
is in the "blue" part of the spectra, corresponding to a Doppler velocity of $-60 \mathrm{~km} \mathrm{~s}^{-1}$. In the "red" part of the spectra, the component with the highest relative intensity appears at a smaller value of Doppler-shift, $\sim 25 \mathrm{~km} \mathrm{~s}^{-1}$.

The initial temperature at the $X$-point for experiment "A2" is about 1.6 times higher than that for experiment "A1", and therefore just slightly below the equilibrium temperature of formation of the $\mathrm{C}$ IV line. As seen in Fig. 2, at the end of the computed time-series in this particular case there is almost no emission left at the rest wavelength. There is only one blue-shifted component present in the spectra, instead of two in the previous time-series relevant to experiment "A1". A very faint red-shifted component at a Doppler velocity of about $20 \mathrm{~km} \mathrm{~s}^{-1}$ is also present. Note how rapidly the central line intensity drops and eventually diminishes in the spectra after $\approx 30 \mathrm{~s}$. The maximum value of the blue-shift is reached at the end of the time-series and is of the order of $-100 \mathrm{~km} \mathrm{~s}^{-1}$. In the "red" part of the spectra, in turn, the largest Doppler-shift is only about $20 \mathrm{~km} \mathrm{~s}^{-1}$.

The central line emission is similarly absent at the end of the time-series relevant to experiment "B2" (the lower right panel in Fig. 2). The initial temperature at the $X$-point for this particular experiment is about twice the equilibrium temperature of formation of the $\mathrm{C}$ IV line. The line-core intensity gradually decreases with time and eventually almost vanishes, whereas two symmetrical Doppler-shifted components progressively develop. After a certain time, these two components become dominant in the spectra. The maximum values of the red- and blueshifts reached at the end of the computed time-series are of the order of $\pm 70 \mathrm{~km} \mathrm{~s}^{-1}$.

In the time-series relevant to experiment "B1" (the lower left panel in Fig. 2), there are no Doppler-shifted components. Instead, there is a continuously broadened emission line with decreasing central intensity. Note in the relevant MHD experiment there is a uniform initial temperature assigned along the line-of-sight, while enhanced mass density (and temperature) is present in the current concentration. In this particular case, the initial temperature at the $X$-point is approximately 2.2 times above the equilibrium temperature of formation of the $\mathrm{C}$ IV line. The relevant ion populations at the locations with fastest moving plasma are lower than those at the locations where the plasma is at rest. Furthermore, because of the initially enhanced mass density in the current concentration, the relevant mass density at the locations of maximum jet velocity substantially decreases as the outflow jets evolve. Note that the emissivity is proportional to the square of the mass density. Taken together, this explains why Doppler-shifted components are missing from the spectra of the C IV line relevant to this particular experiment. Interestingly, we will see that the behavior of the $\mathrm{O}$ VI resonance line is totally different for the same experiment.

One result is clear at this stage. Starting from different initial states, one gets quite different results in computing observable consequences, such as line profiles. This means that the temperature at the $X$-point versus the equilibrium temperature of line formation is critical. Furthermore, the assumption of uniform mass density across the current concentration (experiments "A2" and "B2") affects quite substantially the central line intensity of the C IV line. Since this intensity is far too low compared to that of the Doppler-shifted components, it is very unlikely that the above assumption can be further supported in building a physical model of solar explosive events. This is because such an intensity distribution in the spectra is not confirmed by the relevant observations (see, e.g., Perez et al. 1999; Teriaca et al. 2001).

\subsection{Time series in the $O$ VI $1031.9 \AA$ resonance line}

Results for O vi are shown in Fig. 3. Note that, although line profiles were computed every $0.5 \mathrm{~s}$, the time-series show only those averaged over every approximately $2 \mathrm{~s}$. The upper panels in this figure refer to experiments "A1" (left) and "A2", while the lower ones represent experiments "B1" (left) and "B2", respectively.

The behavior of the O VI line in experiment "A1" is similar to that of the C IV line (see Fig. 2). Note this time the initial temperature at the $X$-point is approximately 3 times below the equilibrium temperature of formation of the resonance $\mathrm{O}$ vi line. Thus, the initial peak population of the O VI ion is again reached somewhere in the hightemperature region towards which the "blue" outflow jet propagates, and away from the $X$-point. One would therefore expect to recognize this jet as a blue-shifted component in the relevant spectra of the $\mathrm{O}$ vi line. This is what Fig. 3 (the upper left panel) actually shows. The "red" jet, however, is not recognized in these time-series. Since the ion populations are strongly dependent on the plasma temperature, it is to be expected that this outflow jet may not be present in the spectra. In turn, there is not only one blue-shifted component seen in Fig. 3 (see also the adjacent image plot), but rather two (or even three). Note that, although the central line intensity gradually decreases with time, there is still line-core emission present in the spectra at the end of the computed time-series. The main blue-shifted component, namely the one which occurs with highest relative intensity, undergoes quite substantial changes in intensity. This first increases with time, reaches a maximum value at about $60 \mathrm{~s}$, and then starts declining. At the end of the time-series, the corresponding Doppler velocity of this blue-shifted component is of the order of $-100 \mathrm{~km} \mathrm{~s}^{-1}$. The other two blue-shifted components appear at lower intensities than the main Doppler component and are best seen at the end of the time-series. The corresponding Doppler velocities of the two faint components are of the order of $-50 \mathrm{~km} \mathrm{~s}^{-1}$ and $-160 \mathrm{~km} \mathrm{~s}^{-1}$, respectively.

The line synthesis for experiment "A2" (the upper right panel in Fig. 3) results in time-series which show almost no emission at the rest wavelength, while there is a strong blue-shifted component present in the spectra. The initial temperature at the $X$-point for this particular 

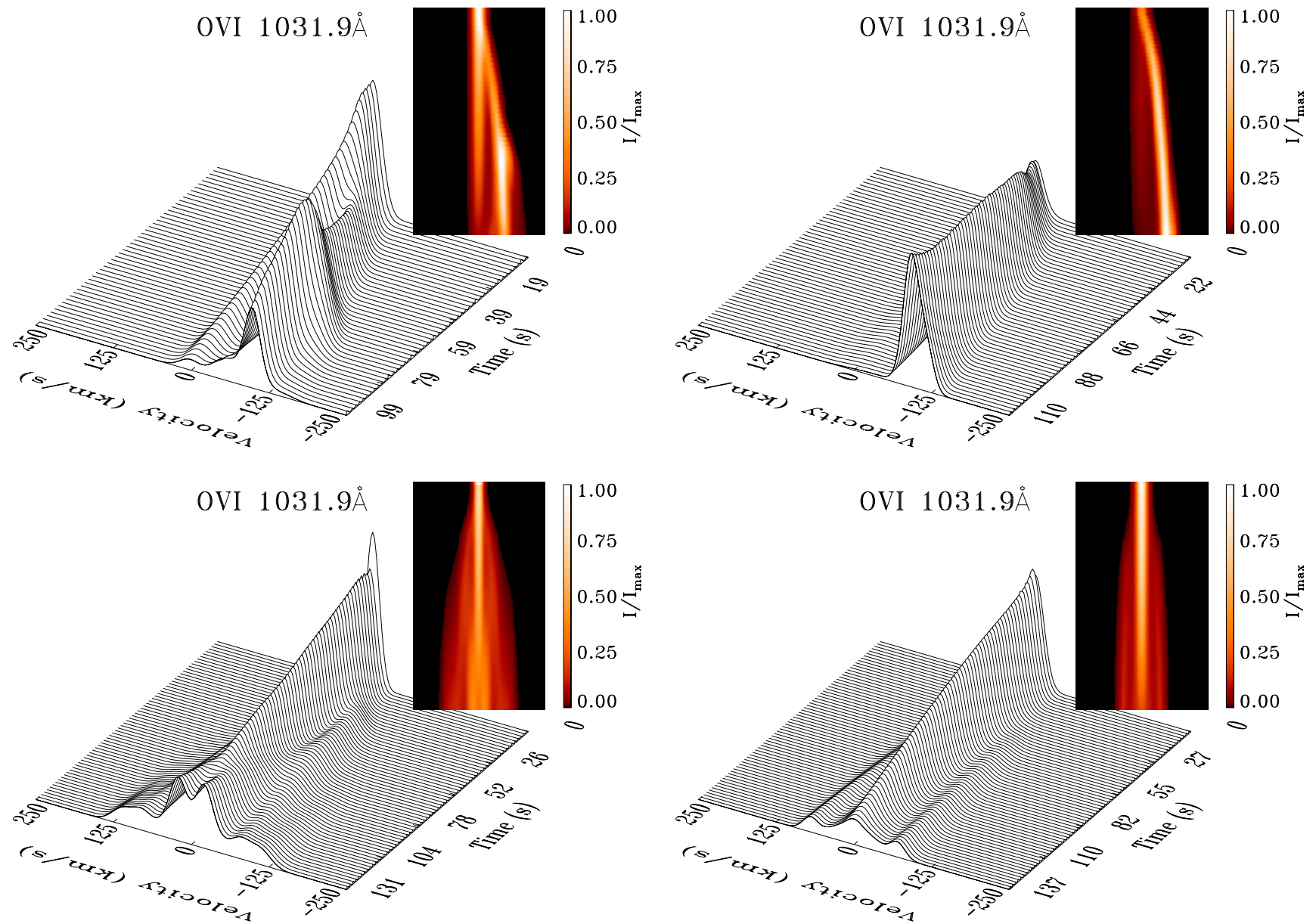

Fig. 3. Line synthesis shown as time-series in the O VI $1031.9 \AA$ resonance line. The upper panels refer to experiments "A1" (left) and "A2", while the lower ones represent experiments "B1" (left) and "B2", respectively. The adjacent image on each panel shows the time-evolution of the spectral line intensity. Note that the time in these images goes from top to bottom (see text for more details).

experiment is about 5 times below the equilibrium temperature of formation of the $\mathrm{O}$ vi line which explains the simulations. This Doppler component occurs at both increased intensity and value of the blue-shift. At the end of the time-series, the latter reaches a maximum value of the order of $-130 \mathrm{~km} \mathrm{~s}^{-1}$.

Simulations for experiment "B2" (the lower right panel in Fig. 3) show both line-core emission and Doppler-shifted components present in the spectra. The relevant MHD experiment assumed uniform mass density both along the line-of-sight and across the current concentration. The corresponding initial temperature at the $X$-point for this particular case is only 1.1 times below the equilibrium temperature of formation of the $\mathrm{O}$ VI line. As seen in the relevant time-series, the central line intensity monotonically decreases with time, while two symmetrical Dopplershifted components eventually occur in the spectra. These components appear at both increasing intensity and absolute value of the Doppler-shift. The maximum value of the latter is reached at the end of the time-series and is of the order of $100 \mathrm{~km} \mathrm{~s}^{-1}$.

A remarkable situation is found for experiment "B1" (the lower left panel in Fig. 3). The corresponding $\mathrm{O}$ VI line profiles undergo an interesting evolution, and reveal signatures very similar to those seen in real observations of solar explosive events. Note that the relevant MHD experiment represents the physical case of uniform initial temperature along the line-of-sight, whereas enhanced mass density is assigned in the current concentration. The initial temperature at the $X$-point for this particular case is approximately 0.7 times the equilibrium temperature of formation of the $\mathrm{O}$ VI line. As seen in Fig. 3, the central line intensity monotonously declines with time (apart from the initial drop seen), while two symmetrical Doppler components gradually develop, which are first recognized at about $t \approx 45 \mathrm{~s}$. At a later stage, two other Dopplershifted components occur in the spectra on each side of the central wavelength. Thus, at the end of the time-series there are six Doppler components present all together. The dominant emission comes from the two symmetrical components with smallest Doppler-shifts. Corresponding Doppler velocities of these components at the end of the time-series are of the order of $\pm 25 \mathrm{~km} \mathrm{~s}^{-1}$. At relatively small intensity, two other components are seen appearing at Doppler-shifts of about $\pm 75 \mathrm{~km} \mathrm{~s}^{-1}$. At intensities slightly fainter than these, two more symmetrical 
components at Doppler-shifts of approximately $\pm 110 \mathrm{~km} \mathrm{~s}^{-1}$ are present. The largest values of blueand red-shifts reached in these time-series are of the order of $\pm 130 \mathrm{~km} \mathrm{~s}^{-1}$. In contrast, relevant simulations for the C IV line show no Doppler components in the spectra, but only continuously broadened emission line with monotonically decreasing line-core intensity with time.

As in the C IV simulations, the initial physical state has a strong impact on the corresponding changes in the spectra. Furthermore, the outflow jets produced by an ongoing magnetic reconnection may be recognized in the spectra of one particular resonance line, while being entirely absent in the spectra of some other lines formed at substantially different temperatures than the former.

\section{Summary}

We examined the response, in terms of observable consequences, of various physical environments resembling the "quiet" Sun atmosphere to a magnetic reconnection event. Our results prove that non-equilibrium ionization must be treated in the numerical modelling of solar transient events. The observable consequences in transition region lines, such as C IV 1548.2 $\AA$ and O VI 1031.9 $\AA$, are strongly dependent on the assumed initial physical state. Furthermore, the visibility of the reconnection events may well be present in one particular resonance line, if appropriate physical conditions are met, while the same events will not be recognized in another emission line. This is because of the difference in the formation temperature between the two lines (e.g., $1 \times 10^{5} \mathrm{~K}$ for $\mathrm{C}$ IV and $3.2 \times 10^{5} \mathrm{~K}$ for $\mathrm{O}$ VI, respectively).

The computed time-series in the two resonance lines revealed quite substantial and complex changes in the relevant spectra, despite the simplicity of the model situations examined. As a general result in both lines, however, those reconnection events appeared as high-velocity events in the relevant time-dependent spectra. Particular assumptions regarding the initial state restrict our ability to compare with observations in these exploratory calculations. For instance, the assumption of uniform mass density across the current concentration (experiments "A2" and "B2" in our notations) affected quite substantially the central line intensity in both lines by being far too low compared to that of the Doppler-shifted components. This is a feature not supported by the relevant observations (see Perez et al. 1999; Teriaca et al. 2001). In turn, initial states involving enhanced mass density in the current concentration (experiments "A1" and "B1" in our notations), may be more appropriate in the physical picture of explosive events. In these circumstances, the line profiles of both lines showed a variety of Doppler-shifted components, together with a line-core emission still being present in the spectra at the end of the computed timeseries.

The value of the length scale, $L_{0}$, also influences the final results, because it defines the time-scale in physical units (through the Alfvén speed, $v_{\mathrm{A} 0}$ ), on which the outflow jets evolve. Furthermore, note that we examined a very particular situation when the symmetry axis of the outflow jets coincides with the line-of-sight. This implies that at other view angles computed line profiles will look different. All this makes the direct comparison of our numerical results with particular observations of explosive events rather difficult, although there is a good overall agreement with the relevant datasets of Perez et al. (1999). Unfortunately, most observational data tend to be limited to individual spectral features. Recent high-cadence observations by Madjarska \& Doyle (2001) obtained in Ly 6 $\left(2 \times 10^{4} \mathrm{~K}\right)$ and $\mathrm{S}$ VI $\left(2 \times 10^{5} \mathrm{~K}\right)$ lines reveal a $\sim 30-40 \mathrm{~s}$ time-delay during explosive events in the response of $\mathrm{S}$ VI with respect to the Ly 6 line, with the event being seen first in Ly 6. Further spectroscopic observations simultaneously covering lines with a wide range of formation temperature obtained with high-temporal resolution are required to determine the time-evolution of the physical conditions involved in solar explosive events. We believe the results presented here provide some of the essential physical insight needed to assess the meaning of observations in terms of physical properties, although these should perhaps be considered more as numerical experiments rather than simulations.

Acknowledgements. Research at Armagh Observatory is grantaided by the N. Ireland Dept. of Culture, Arts and Leisure. The National Center for Atmospheric Research is sponsored by the National Science Foundation. This work was partly supported by a grant from the British Council - Acciones Integradas Program (Spain) ref. No. 1814. and PPARC grants PPA/G/S/1999/00055 \& PPA/V/S/1999/00668. I. Roussev dedicates his studies to Ana and Elena. K. Galsgaard and I. Roussev are grateful for the warm hospitality they received during their visits at Space \& Atmosphere Research Center (SPARC), Department of Applied Mathematics, University of Sheffield, where part of this work was prepared. I. Roussev is also thankful to the Department of Applied Mathematics, University of St. Andrews, and the NSO in Tucson, Arizona, for the support provided during his visits there. We are also thankful to P. G. Judge, B. C. Low, and the anonymous referee for the useful suggestions made to improve the quality of this paper.

\section{References}

Arnaud, M., \& Rothenflug, R. 1985, A\&AS, 60, 425

Madjarska, M. S., \& Doyle, J. G. 2001, A\&A, in press

Perez, M. E., Doyle, J. G., Erdélyi, R., \& Sarro, L. 1999, A\&A, 342,279

Roussev, I., Galsgaard, K., Erdélyi, R., \& Doyle, J. G. 2001a, A\&A, 370, 298

Roussev, I., Galsgaard, K., Erdélyi, R., \& Doyle, J. G. 2001b, A\&A, 375, 228

Sarro, L. M., Erdélyi, R., Doyle, J. G., \& Perez, E. P. 1999, A\&A, 351, 721

Summers, H. P. 1972, MNRAS, 158, 255

Teriaca, L., Madjarska, M. S., \& Doyle, J. G. 2001, Sol. Phys., 200, 91 